Miami Nature Biotechnology Short Reports

TheScientificWorld (2001) 1 (S3), 130SR

ISSN 1532-2246; DOI 10.1100/tsw.2001.223

\title{
IDENTIFICATION OF RENOX, A NOVEL SUPEROXIDE PRODUCING ENZYME IN KIDNEY
}

\author{
Miklós Geiszt and Thomas L. Leto* \\ Laboratory of Host Defenses, NIAID, National Institutes of Health \\ *tleto@nih.gov
}

INTRODUCTION. Reactive oxygen species (ROS) have a crucial role in diverse physiological and pathological processes. ROS production by phagocytic cells is essential for normal host defense (1) while ROS production by nonimmune cells has important roles in apoptosis, mitogenic responses and oxygen sensing $(2,3)$. In phagocytic cells superoxide is produced by the NADPH oxidase enzyme complex that transfers electrons from NADPH to molecular oxygen (1). The electron-transporting component of the NADPH-oxidase is gp91 ${ }^{\text {phox }}$, a glycosylated flavoprotein that forms a hetero-dimer with $\mathrm{p} 22^{\mathrm{phox}}$.

We have identified novel gp91 ${ }^{\text {phox }}$ homolog in kidney referred to as a renal oxidase or Renox, which is highly expressed in renal tubular cells and may fulfill the function of the putative oxygen sensor in the kidney.

METHOD. Full-length murine Renox cDNA was isolated by 5`and 3` RACE from mouse kidney RNA as a template for cDNA synthesis. Gene expression was analyzed by Northern blot and in situ hybridization. Functional studies (superoxide production and proliferation) were performed by transfecting NIH 3T3 cells with the Renox cDNA.

RESULTS. The murine Renox is a 578 amino acid long protein showing $40 \%$ sequence identity and $57 \%$ similarity to mouse gp91 ${ }^{\text {phox }}$ while the human homolog is also a 578 amino acid long protein with $90 \%$ identity to its mouse counterpart. Renox contains conserved features considered critical for NADPH oxidase function, namely six hydrophobic segments within the amino-terminal portion, proposed as membrane-embedded domains involved in transmembrane electron transport, as well as sequence motifs corresponding to proposed binding sites for heme, flavin, and NADPH.

Northern blot experiments revealed that Renox is highly expressed in the kidney. In situ hybridization experiments on mouse kidney sections demonstrated that Renox is present in proximal tubular cells of the kidney while in human kidney inner medulary collecting duct cells show strong expression.

Renox-transfected cells showed increased superoxide production when compared with control lines and Renox-transfected fibroblasts showed drastic changes in cellular morphology and a decreased rate of proliferation.

DISCUSSION. Based on its expression in the proximity of erythropoietin production and its demonstrated capacity for production of superoxide we propose that Renox is a likely candidate for the oxygen sensor function in kidney. 


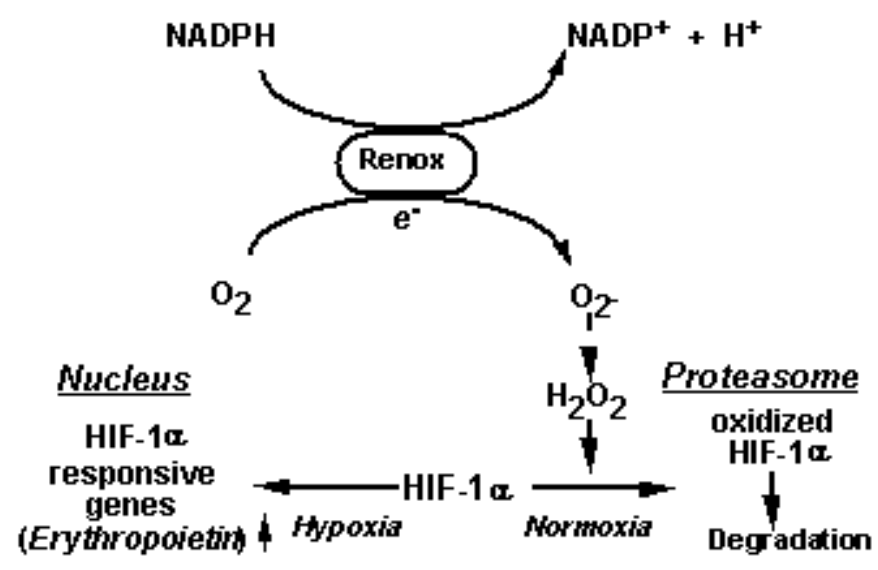

Fig. 1. Proposed scheme for down-regulation of erythropoietin production by Renox-generated ROS.

\section{REFERENCES.}

1. Leto, T.L. (1999) in Inflammation Basic Principles and Clinical Correlates (Gallin, J.I. and Snyderman, R., Ed.) Lippincott Williams \&Wilkins, Philadelphia, pp. 769-787

2. $\quad$ Ebert, B.L. and Bunn, H.F. (1999) Blood 94, 1864-1877

3. $\quad$ Finkel, T. and Holbrook, N.J. (2000) Nature 408, 239-247 

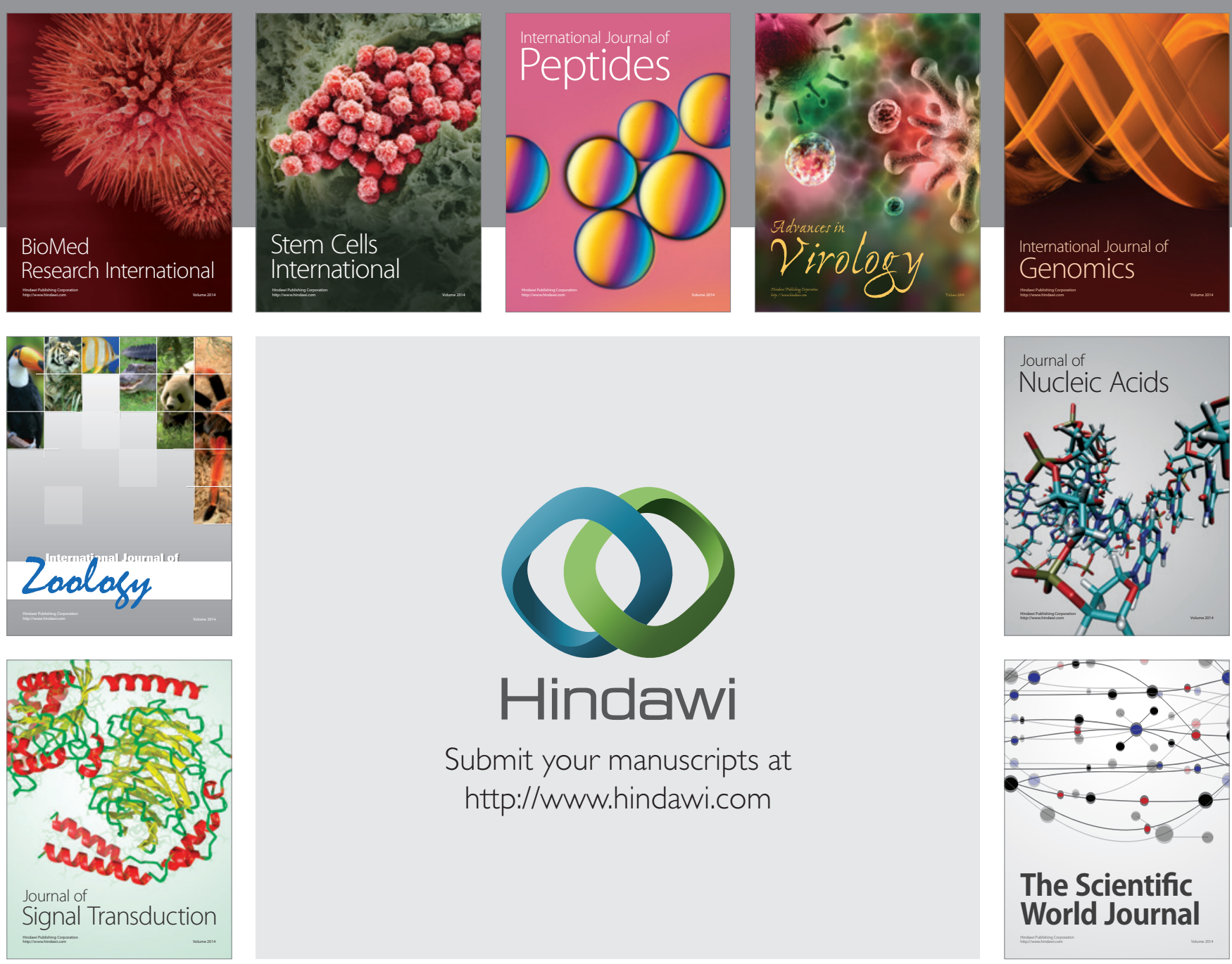

Submit your manuscripts at

http://www.hindawi.com
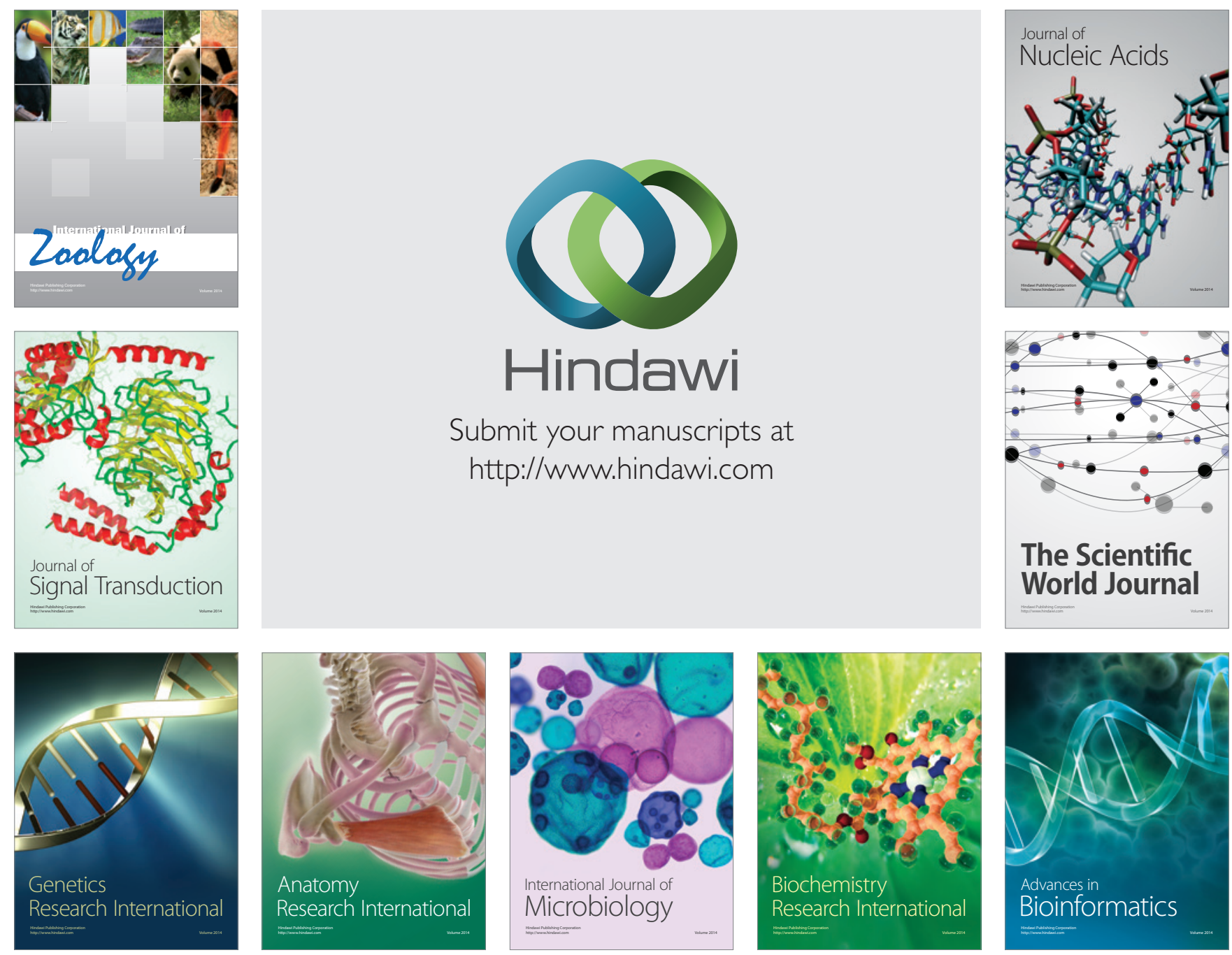

The Scientific World Journal
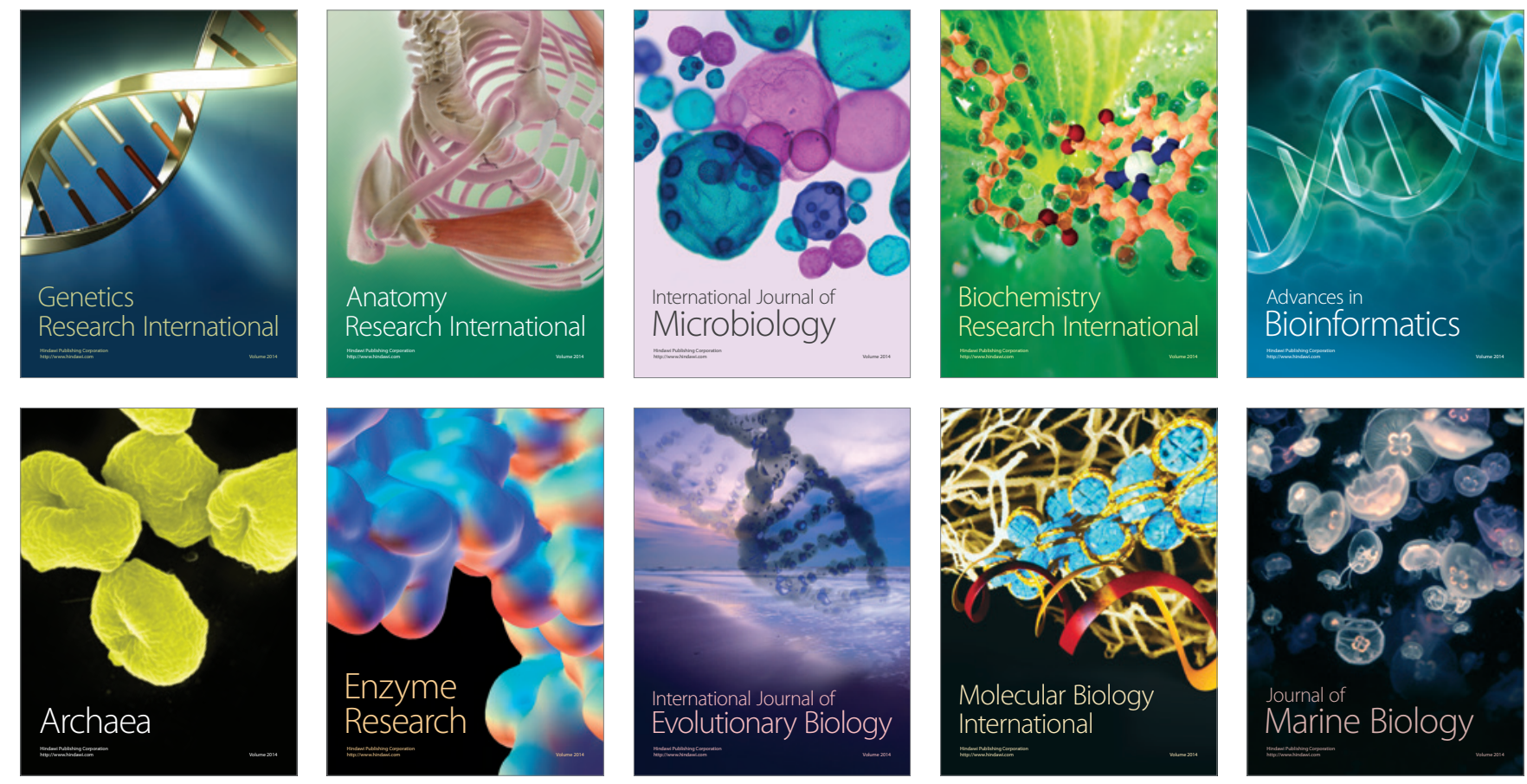